\title{
The conceptual framework for SARS-CoV-2 related lymphopenia
}

Ilnaz Rahimmanesh ${ }^{1}$, Shirin Kouhpayeh ${ }^{2}$, Hossein Khanahmad $^{1 *}$

1- Department of genetics and molecular biology, School of Medicine, Isfahan University of Medical Sciences, Isfahan, Iran

2- Department of Immunology, Erythron Genetics and Pathobiology Laboratory, Isfahan, Iran

\section{*Corresponding author: Dr. Hossein Khanahmad}

Isfahan University of Medical Sciences, School of Medicine, Department of Genetics and Molecular Biology, Isfahan, Iran.

Phone: +98-313-7929144

Fax number: +98-313-6688597

H_khanahmad@med.mui.ac.ir

ABSTRACT: 
The emerging of severe acute respiratory syndrome coronavirus 2 (SARS-CoV-2) outbreak is associated with high morbidity and mortality rates globally. One of the most prominent characteristics of coronavirus disease-19 (COVID-19) is lymphopenia which is in contrast to other viral infections. This controversy might be explained by the evaluation of impaired innate and adaptive immune responses during the SARS-CoV-2 infection. During the innate immune response, poly-ADP-ribose polymerase (PARP) hyperactivated due to virus entry and extensive DNA damage sequentially leading to NAD+ depletion, ATP depletion and finally cell death. In contrast to the immune response against viral infections, cytotoxic T lymphocytes decline sharply in SARS-CoV-2 infection which might be due to infiltration and trapping in the lower respiratory tract. In addition, there are more factors proposed to involve in lymphopenia in COVID-19 infection like the role of CD38 which functions as NADase and intensifies NAD depletion which in turn affects NAD+ dependent Sirtuin proteins, as the regulators of cell death and viability. Lung tissue sequestration following cytokine storm supposed to be another reason for lymphopenia in COVID-19 patients. Protein 7a as one of the virus-encoded proteins induces apoptosis in various organ-derived cell lines. These mechanisms proposed to induce lymphopenia, although there are still more studies needed to clarify the underlying mechanisms for lymphopenia in COVID-19 patients.

Key words: SARS-CoV-2, lymphopenia, NAD+ depletion, CD38

\section{Introduction:}

The coronavirus disease 19 (COVID-19), a novel infectious disease, caused by severe acute respiratory syndrome coronavirus 2 (SARS-CoV-2), which originated in Wuhan, China, has rapidly increased in pandemic scale with growing morbidity and mortality rate worldwide. In the 
past decades, two coronavirus family members were responsible for severe respiratory disease outbreaks which have been previously characterized as a major concern of public health; severe acute respiratory syndrome (SARS) and Middle East respiratory syndrome (MERS) caused by SARS and MERS coronavirus, respectively (1).

Coronaviruses spread broadly among humans leading to a spectrum of respiratory diseases ranging from flu-like symptoms or pneumonia to acute respiratory distress syndrome (ARDS) which caused high mortality in human populations (2).

Although the pathogenesis of SARS-CoV-2 is not yet fully understood, there are apparent similarity between SARS-CoV-2 and the original SARS-CoV. Extensive lung damage in COVID19 patients appears to be associated with high initial viral load, increased monocyte, macrophage and neutrophil infiltration in the lungs concomitant with elevated levels of serum proinflammatory cytokines and chemokines and a rapid decrease of peripheral $\mathrm{T}$ cell subsets (3). Therefore, the clinical deterioration and tissue damage during SARS-CoV-2 infection may result from a combination of direct virus-induced cytopathic effects and maladjusted immune responses. As described earlier, one of the clinical features of SARS-CoV-2 infection is the global reduction of peripheral T cell subsets as a unique characteristic in COVID-19 patients during acute infection (4). Recent data shed new light on the role of impaired immune response and eventual lymphopenia in SARS-CoV-2 pathogenesis (5). Here, we provide a brief introduction to immune response against SARS-CoV-2 and possible mechanisms underlying reduced peripheral $\mathrm{T}$ cell subsets in COVID-19 patients.

\section{The innate immune response}

The SARS-CoV-2 spike protein has a strong binding affinity for human angiotensin II receptor (ATR1) which considered essential for host cell entry and subsequent viral progeny. During an 
acute respiratory infection, the innate immune response is the first line of defense against the virus leading to initiation of a rapid immune response following virus-cell interaction (6).

In the conventional cell biology, ADP-ribosylation is a common reversible posttranslational modification with proposed antiviral properties and impact on innate immunity. ADP-ribosylation is mediated by poly-ADP-ribose polymerase (PARP) gene family encoded proteins. Transfer of one or more ADP-ribose (ADPr) groups from nicotinamide adenine dinucleotide (NAD+) to target protein is catalyzed within eukaryotic cells by members of the PARP, now called as Diphtheria toxin-like ADP-Ribosyltransferases (ARTDs) (7, 8). PARP-1 hub role is sensing cellular metabolic stress including oxidative stress, DNA repair, and pathogen infection which lead to activation and ultimately determination of cell fate. The PARPs activity is mainly correlated with the regulation of the mammalian innate antiviral response (9-11). Since the NAD+ level is critical for regulation of energy metabolism and maintenance of redox homeostasis, PARP hyperactivation following extensive DNA damage upon viral infection results in rapid depletion of cellular NAD+ and reduced ATP production and ultimately cell death $(12,13)$.

\section{The adaptive immune response:}

Adaptive immune response against viral infections started to develop a precise and powerful protector immunity against viruses. The adaptive immune response to viral infections exerts through the effector function of cytotoxic T lymphocyte (CTL) response (14). CTLs are generated in response to intracellular invading pathogens, and they specifically recognize and kill virusinfected cells and/or release inhibitory antiviral soluble factors. Thus, a sharp increase in CTL count is highly expected in CTL patients with SARS-CoV-2 infection. 
However, unlike the conventional immune responses against viruses, in SARS-CoV-2 infection a different response shaped which is accompanied with particular T cell lymphopenia, with a rapid decrease in both CD4 and CD8 T cell subsets (4-6).

The latest report, indicated the reduced number of CD4+ and CD8+ T cells in the peripheral blood of SARS-CoV-2-infected patients, similar to patients with SARS-CoV, with concomitant hyperactivated T cells shown by high proportions of CD4-restricted HLA-DR (3.47\%) and CD38 (CD8 39.4\%) double-positive populations(15) .

The possible explanation of CTL reduction in COVID-19 patients might be an adverse outcome which need further investigations. Infiltration of $\mathrm{T}$ cells and subsequent trapping in the lower respiratory tract as well as immune cell death could also participate in the underlying mechanisms of the acute decrease of peripheral $\mathrm{T}$ cell subsets observed in SARS-CoV-2 infection.

Here, the possible factors which might be involved in general reduction of peripheral $\mathrm{T}$ lymphocytes during SARS-CoV-2, are further discussed in details.

\section{CD38 expression:}

Expression of CD38 on immune system cells seems to play an important role in the context of host defense to infection (16).

Apart from being a cell surface receptor, CD38 is a transmembrane glycoprotein with bifunctional activity of both ADP-ribosyl cyclase and cyclic ADP-ribose hydrolase CD38 utilizes NAD(P) as a substrate to produce the second messengers, Nicotinic acid adenine dinucleotide phosphate (NAADP) and Cyclic adenosine diphosphate ribose (cADPR) by mean of ADP-ribosyl cyclase activity. It has been postulated that CD38 is the major NADase in cells and NAD levels 
significantly increased upon CD38 knock down (17). Thus, it was hypothesized that NAD depletion upon CD38 overexpression is associated with disturbed metabolic regulation and cell viability. NAD+ known as one of the energy currencies required for vital cellular processes mediating bio- energetic processes, metabolic homeostasis, response to damages and immune reactions. Moreover, emerging evidences demonstrated NAD+ is released during the early phase of inflammation and exerts the immunoregulatory role in vivo $(18,19)$.

As part of the innate immune response, PARP hyper-activation, subsequent to viral mediatedoxidative and/or nitrosative stress, consumes large amounts of NAD+ and CD38 overexpression during adaptive immunity, culminates in NAD+ depletion. The NAD+ depletion linked with a reduced glycolytic activity which may, in turn, affect ATP levels, since cells consume ATP for NAD+ replenishment. On the other hand, NAD+ depletion leads to increased production and release of pro-inflammatory cytokines, reactive oxygen species, and macrophage infiltration via Sirtuin-1 (SIRT1) inhibition $(8,20)$.

Proteins of the SIRT family (sirtuins) are NAD+-dependent histone deacetylases (HDAC) which govern the balance between cellular durability and death. SIRT proteins are thought to exert their function through the control of genomic stability, DNA repair and transcriptional regulation. In addition to SIRT proteins, PARP operates convergent with SIRT proteins for maintenance of the balance that determines cell fate in response to stress (21).

The functional cross-talk between SIRT proteins and PARP is suggested following the consumption of the common intracellular NAD+ pool by both of them. The previous results showed over-activation of PARP subsequent to DNA damage leads to AIF (Apoptosis Inducing Factor)-mediated cell death in the absence of $\operatorname{SIRT1}(20,22)$. 


\section{Pro-inflammatory cytokines and chemokines:}

Patients infected with COVID-19 showed significantly increased levels of plasma proinflammatory cytokines including MCP1, MIP1 $\alpha$, MIP1 $\beta$, IL1- $\beta$, IL1RA, IL7, IL8, IL9, IL10, IP10, PDGFB basic FGF2, GCSF, GMCSF, IFN $\gamma$, TNF $\alpha$, and VEGFA $(3,15)$.

This cytokine profile indicates a rapid recruitment and increased trafficking of the monocytemacrophage lineage into the lung very early in the infection process. The immune cell infiltration into the lower respiratory tract, leading to uncontrolled immune responses with subsequent hyperinflammation and cytokine storm results in organ failure, pulmonary tissue damage, and reduce lung capacity $(14,23)$. Thus, tissue sequestration might play the major role in reduction of peripheral blood lymphocyte count of SARS-CoV-2 infected patients.

\section{Protein 7a induced apoptosis:}

The lack of angiotensin converting enzyme II receptor as the SARS-CoV-2 specific receptor, in T and B lymphocytes and macrophages of all haemato-lymphoid organs proposes that direct viral infection is not the reason of acute lymphopenia in SARS-CoV-2 infection (24).

Among the virus-encoded proteins, protein 7a, a structural protein specifically encoded by SARS$\mathrm{CoV}-2$, combine to mature virions and plays an essential role in the pathogenesis of SARS-CoV2 (25). This protein induces apoptosis, arrests the cell cycle, and promotes the production of proinflammatory cytokines. Overexpression of protein 7a, found to induce apoptosis via a caspasedependent pathway in cell lines derived from various organs, including lung, liver and kidney and could be considered as one of the mechanisms involved in lymphopenia (26).

\section{Conclusion:}


In conclusion, the innate and adaptive immune responses against SARS-CoV-2 and possible mechanisms involved in reduction of peripheral lymphocyte subsets in patients with SARS-CoV2 was discussed. Although, the precise mechanism underlying the acute lymphopenia during SARS-CoV-2 infection remains unclear and further researches is vital for identification of molecular mechanisms responsible for the pathogenesis of SARS-CoV-2 infection.

1. Guo Y, Korteweg C, McNutt MA, Gu J. Pathogenetic mechanisms of severe acute respiratory syndrome. Virus research. 2008;133(1):4-12.

2. Lau YL, Peiris JS. Pathogenesis of severe acute respiratory syndrome. Current opinion in immunology. 2005;17(4):404-10.

3. Li G, Fan Y, Lai Y, Han T, Li Z, Zhou P, et al. Coronavirus infections and immune responses. Journal of medical virology. 2020;92(4):424-32.

4. Cui W, Fan Y, Wu W, Zhang F, Wang J-y, Ni A-p. Expression of lymphocytes and lymphocyte subsets in patients with severe acute respiratory syndrome. Clinical infectious diseases. 2003;37(6):857-9.

5. Li T, Qiu Z, Zhang L, Han Y, He W, Liu Z, et al. Significant changes of peripheral T lymphocyte subsets in patients with severe acute respiratory syndrome. The Journal of infectious diseases. 2004;189(4):64851.

6. Li X, Geng M, Peng Y, Meng L, Lu S. Molecular immune pathogenesis and diagnosis of COVID-19. Journal of Pharmaceutical Analysis. 2020.

7. Fouquerel E, Sobol RW. ARTD1 (PARP1) activation and NAD(+) in DNA repair and cell death. DNA repair. 2014;23:27-32.

8. Graeff R, Liu Q, Kriksunov IA, Kotaka M, Oppenheimer N, Hao Q, et al. Mechanism of cyclizing NAD to cyclic ADP-ribose by ADP-ribosyl cyclase and CD38. The Journal of biological chemistry. 2009;284(40):27629-36.

9. Grady SL, Hwang J, Vastag L, Rabinowitz JD, Shenk T. Herpes simplex virus 1 infection activates poly (ADP-ribose) polymerase and triggers the degradation of poly (ADP-ribose) glycohydrolase. Journal of virology. 2012;86(15):8259-68. 
10. Liu L, Su X, Quinn WJ, 3rd, Hui S, Krukenberg K, Frederick DW, et al. Quantitative Analysis of NAD Synthesis-Breakdown Fluxes. Cell metabolism. 2018;27(5):1067-80.e5.

11. Siegel C, McCullough LD. NAD+ depletion or PAR polymer formation: which plays the role of executioner in ischaemic cell death? Acta physiologica (Oxford, England). 2011;203(1):225-34.

12. Adriouch S, Hubert S, Pechberty S, Koch-Nolte F, Haag F, Seman M. NAD\&It;sup\&gt;+\&It;/sup\&gt; Released during Inflammation Participates in T Cell Homeostasis by Inducing ART2-Mediated Death of Naive T Cells In Vivo. The Journal of Immunology. 2007;179(1):186.

13. Hsieh C-L, Hsieh S-Y, Huang H-M, Lu S-L, Omori H, Zheng P-X, et al. Nicotinamide Increases Intracellular NAD+ Content to Enhance Autophagy-Mediated Group A Streptococcal Clearance in Endothelial Cells. Frontiers in Microbiology. 2020;11(117).

14. Chen J, Lau YF, Lamirande EW, Paddock CD, Bartlett JH, Zaki SR, et al. Cellular Immune Responses to Severe Acute Respiratory Syndrome Coronavirus (SARS-CoV) Infection in Senescent BALB/c Mice: CD4\&lt;sup\&gt;+\&lt;/sup\&gt; T Cells Are Important in Control of SARS-CoV Infection. Journal of Virology. 2010;84(3):1289.

15. Xu Z, Shi L, Wang Y, Zhang J, Huang L, Zhang C, et al. Pathological findings of COVID-19 associated with acute respiratory distress syndrome. The Lancet respiratory medicine. 2020.

16. Camacho-Pereira J, Tarragó MG, Chini CCS, Nin V, Escande C, Warner GM, et al. CD38 Dictates Age-Related NAD Decline and Mitochondrial Dysfunction through an SIRT3-Dependent Mechanism. Cell Metab. 2016;23(6):1127-39.

17. Chini EN. CD38 as a regulator of cellular NAD: a novel potential pharmacological target for metabolic conditions. Curr Pharm Des. 2009;15(1):57-63.

18. Chini CCS, Tarrago MG, Chini EN. NAD and the aging process: Role in life, death and everything in between. Molecular and cellular endocrinology. 2017;455:62-74.

19. Johnson S, Imai S-I. NAD (+) biosynthesis, aging, and disease. F1000Res. 2018;7:132-.

20. Canto C, Sauve AA, Bai P. Crosstalk between poly(ADP-ribose) polymerase and sirtuin enzymes. Molecular aspects of medicine. 2013;34(6):1168-201.

21. Aksoy P, Escande C, White TA, Thompson M, Soares S, Benech JC, et al. Regulation of SIRT 1 mediated NAD dependent deacetylation: a novel role for the multifunctional enzyme CD38. Biochem Biophys Res Commun. 2006;349(1):353-9.

22. Kolthur-Seetharam U, Dantzer F, McBurney MW, Murcia Gd, Sassone-Corsi P. Control of AIFmediated Cell Death by the Functional Interplay of SIRT1 and PARP-1 in Response to DNA Damage. Cell Cycle. 2006;5(8):873-7.

23. To KF, Lo AW. Exploring the pathogenesis of severe acute respiratory syndrome (SARS): the tissue distribution of the coronavirus (SARS-CoV) and its putative receptor, angiotensin-converting enzyme 2 (ACE2). The Journal of pathology. 2004;203(3):740-3.

24. Schoeman D, Fielding BC. Coronavirus envelope protein: current knowledge. Virology journal. 2019;16(1):69.

25. Nieto-Torres JL, DeDiego ML, Verdia-Baguena C, Jimenez-Guardeno JM, Regla-Nava JA, Fernandez-Delgado R, et al. Severe acute respiratory syndrome coronavirus envelope protein ion channel activity promotes virus fitness and pathogenesis. PLoS pathogens. 2014;10(5).

26. DeDiego ML, Nieto-Torres JL, Jiménez-Guardeño JM, Regla-Nava JA, Alvarez E, Oliveros JC, et al. Severe acute respiratory syndrome coronavirus envelope protein regulates cell stress response and apoptosis. PLoS pathogens. 2011;7(10). 